\title{
Efecto de la temperatura y salinidad en la mortalidad de adultos de Capitella sp. (Polychaeta: Capitellidae) en el laboratorio
}

\section{Melissa Herrera-Perez ${ }^{1,2}$ \& Nuria Méndez ${ }^{3}$}

1. Laboratorio de Ecotoxicología Acuática. Área Funcional de Investigaciones Marino Costeras (AFIMC) de la Dirección General de Investigaciones en Acuicultura (DGIA). Instituto del Mar del Perú (IMARPE). Callao, Perú; imelissa.herrera.p@gmail.com

2. Facultad de Ciencias Biológicas. Universidad Ricardo Palma (URP). Santiago de Surco, Lima, Perú.

3. Unidad Académica Mazatlán. Instituto de Ciencias del Mar y Limnología, Universidad Nacional Autónoma de México. P.O. Box 811, Mazatlán 82000, Sinaloa, México; nuri@ola.icmyl.unam.mx

$$
\text { Recibido 11-VIII-2018. C Corregido 06-II-2019. Aceptado 30-VI-2019. }
$$

\begin{abstract}
Effect of temperature and salinity on mortality of adults of Capitella sp. (Polychaeta: Capitellidae) under laboratory conditions. Introduction: The marine-estuarine polychaete Capitella capitata is an endobenthic species which is considered as a cosmopolitan organism. The laboratory culture of this species-complex allows its use as a test species in toxicity bioassays and reproduction studies. Objective: It is pretended to observe mortality under two temperature and salinity ranges as optimal conditions to culture Capitella sp. in the laboratory. Metodology: Polychaetes were collected in Callao Province, Peru, during November 2016 and January 2017. A bioassay was carried out to observe mortality under different temperature and salinity ranges using two treatments: treatment 1 , with polychaetes collected during January 2017, which were subjected to conditions similar to the collection site (temperature: $20-22^{\circ} \mathrm{C}$, salinity: $30-32 \mathrm{psu}$ ); treatment 2 , with the organisms collected during November 2016 , with temperature $\left(24-26^{\circ} \mathrm{C}\right)$ and salinity $(32$ a 34 ups) selected according to bibliographic data. Results: The increase of temperature and time significantly influenced polychaete mortality of the two treatments (Spearman correlation), while salinity and dissolved oxygen did not show correlation with mortality. Significant effect of time was observed on mortality, with more deaths from the second half of the experiment. Organisms from treatment 1, with environmental conditions similar to those of their habitat survived more than worms from treatment 2 . These results were also observed in the porcentual varibility of the variation coefficient among days in each treatment. The cumulative mortality curve did not show differences between treatments. Conclusion: The optimal ranges to culture Capitella sp. in the laboratory are those from their habitat. In addition to temperature and time, other posible causes of the observed mortality can be worms age, health or maturity, and probably the bad quality of the water used during the bioassay.
\end{abstract}

Key words: Polychaetes, survivorship, bioassay, temperature, salinity, dissolved oxygen.

Herrera-Perez, M., \& Méndez, N. (2019). Efecto de la temperatura y salinidad en la mortalidad de adultos de Capitella sp. (Polychaeta: Capitellidae) en el laboratorio. Revista de Biología Tropical, 67(S5) Suplemento, S51-S62.

Los anélidos poliquetos son gusanos anillados que juegan un papel importante en la ecología de comunidades marinas, debido a la diversidad de sus estructuras y funciones (Olive, 1994). Los poliquetos cada vez tienen más importancia comercial en la industria de la acuicultura como alimento vivo de varias especies cultivables. Algunas especies de organismos vivos pueden estimular la maduración de las gónadas de varias especies comerciales de peces o crustáceos que se cultivan en criaderos (Serebiah, 2015). Otro punto importante 
del cultivo de poliquetos es su uso como cebo, ya que favorece la reducción de los disturbios en los sedimentos y el gran impacto biogeoquímico y el producido por las comunidades bentónicas (Gambi, Castelli, Giangrande, Lanera, Prevedelli, \& Vandini, 1994). Algunos poliquetos de tallas pequeñas son generalmente r-estrategas, tales como algunos espiónidos y capitélidos, son también utilizados en acuacultura como suplemento de la dieta de peces y crustáceos de interés comercial (Gambi et al., 1994). Las técnicas de cultivo de las diferentes especies de poliquetos varían dependiendo de los hábitos y tamaño de las especies, pero el control de la temperatura del agua es primordial (Olive, 1999). Asimismo, es recomendable controlar, además de la temperatura, la salinidad y el tipo de sedimento, este último, debido a que los sedimentos de tamaño de grano pequeño acumulan materia orgánica que puede producir fluctuaciones en los niveles de oxígeno disuelto (Fidalgo e Costa, Passos, \& Cancela da Fonseca, 2003; Méndez, 2015).

El poliqueto marino y estuarino Capitella capitata (Fabricius, 1780) es una especie endobentónica (Tomassetti \& Porrello, 2005) y considerado cosmopolita (Grémare, Marsh, \& Tenore, 1989; Halanych \& Borda, 2009). Este poliqueto es un depositívoro, y se le considera una especie oportunista por su rápido crecimiento, corto ciclo de vida y alta fecundidad (Eckelbarger, Linley, \& Grassle, 1984; Horng, Wang, \& Cheng, 2009; Pardo, Teixeira, \& Amaral, 2010). Ha sido denominado indicador universal de contaminación orgánica en sedimentos marinos debido a su alta tolerancia a las condiciones anóxicas presentes en sedimentos enriquecidos (Reish \& Martin, 1976; Warren, 1976; Gamenick, Vismann, \& Grieshaber, 1998; Linke-Gamenick, Vismann, \& Forbes, 2000a; Méndez, 2005). Capitella capitata constituye un complejo de al menos 50 especies crípticas (Blake, Grassle, \& Eckelbarger, 2009) que se diferencian en su modo reproductivo (e.g., desarrollo de su ciclo de vida, temporada reproductiva, tamaño de los huevos y capacidad de dispersión larvaria; (Tsutsumi \& Kikuchi, 1984; Eckelbarger \& Grassle; 1987,
Méndez, 1995; Méndez, Anguas-Cabrera, \& García-de la Parra, 2008). Asimismo, existen diferencias en las características ecofisiológicas, tolerancia a factores abióticos y condiciones de desoxigenación (Gamenick et al., 1998; Linke-Gamenick et al, 2000a).

Capitella capitata ha sido utilizado como alimento de juveniles del pez Pseudopleuronectes americanus en sistemas integrados de policultivo para el tratamiento de los desechos provenientes de la acuicultura (Ryther et al., 1975). Diferentes estudios señalan que el cultivo y reproducción de algunas especies crípticas del complejo de Capitella es relativamente sencillo debido a su gran abundancia en sedimentos enriquecidos con materia orgánica, sus ciclos de vida cortos, su crecimiento rápido y por la facilidad para su recolección en el campo, su transporte y su mantenimiento en laboratorio (Méndez \& Green-Ruiz, 2006; Dean, 2008; Méndez \& Barata, 2015). El cultivo en el laboratorio de este complejo de especies permite su utilización como especie para pruebas en bioensayos de toxicidad, así como para estudios de reproducción (Reish \& Gerlinger, 1997; Méndez, 2015).

Los estudios de Polychaeta en el Perú se restringen a nivel taxonómico, ecológico y de biodiversidad. Sin embargo, este es el grupo menos estudiado en comparación con otros invertebrados como moluscos, crustáceos y equinodermos, entre otros (Aguirre \& Canales, 2017). El presente trabajo pretende observar la mortalidad en dos rangos de temperatura y salinidad como condiciones óptimas para cultivar Capitella sp. en el laboratorio. Esta información permitirá realizar estudios reproductivos para su utilización como especies de prueba en estudios ecológicos, ecotoxicológicos, biotecnológicos, alimenticios y reproductivos.

\section{MATERIALES Y MÉTODOS}

Recolección de organismos: Individuos de Capitella sp. se recolectaron en La Poza de la Arenilla, Malecón Pardo, distrito de La Punta, en la Provincia Constitucional del Callao (1204'24.1" S - 7709'38.2" W) en noviembre 
de 2016 y enero de 2017. El sedimento superficial (15 cm de profundidad) se recolectó con una espátula de aluminio y se tamizó con una malla de $500 \mu \mathrm{m}$. El material biológico retenido en el tamiz se colocó en envases de plástico de $500 \mathrm{~mL}$ con agua de mar para su traslado al laboratorio. En noviembre (primavera) la temperatura superficial promedio del mar fue $26.5^{\circ} \mathrm{C}$, la salinidad de 32.2 ups y el oxígeno disuelto de $5.04 \mathrm{~mL} / \mathrm{L}$, mientras que en enero (verano) el promedio de temperatura fue $22.6^{\circ}$ C, la salinidad de 29.6 ups y el oxígeno disuelto de $8.39 \mathrm{~mL} / \mathrm{L}$. Estas mediciones se obtuvieron con un equipo multiparamétrico portátil (Hach HQ40D).

En el laboratorio, los especímenes fueron identificados utilizando un microscopio estereoscopio (Olympus SZ-61 ILST 1K36156). Para la identificación de los especímenes de Capitella sp. se siguieron las claves de Fauchald (1977) y Blake (2009).

Condiciones de laboratorio: Para el mantenimiento de los especímenes en el laboratorio se acondicionaron mesas de enfriamiento reguladas a $25^{\circ} \mathrm{C}$. Se utilizaron dos vasos de precipitados de $1 \mathrm{~L}$ de capacidad y en cada uno se colocó una capa de 2 a $4 \mathrm{~cm}$ de sedimento experimental (Linke-Gamenick, Forbes, \& Méndez, 2000b; Méndez, 2005; Méndez, 2006b), $1000 \mathrm{~mL}$ de agua de mar filtrada (< $1 \mu \mathrm{m})$ con salinidad de 35 ups (procedente de las cisternas para el cultivo de conchas de abanico) y 200 individuos de Capitella sp. para el bioensayo. El periodo de aclimatación a las condiciones de laboratorio duró dos semanas (Kenny, 1969).

El ensayo sobre la mortalidad de adultos de Capitella sp. en condiciones de laboratorio se realizó en el Laboratorio de Ecotoxicología Acuática, Área Funcional de Investigaciones Marino Costeras (AFIMC) de la Dirección General de Investigaciones en Acuicultura (DGIA) del Instituto del Mar del Perú (IMARPE), Provincia Constitucional del Callao, Perú.

Sedimento experimental: El sedimento que se utilizó en el ensayo, se recolectó en el área de estudio, junto con las muestras biológicas, y se transportó al laboratorio en bolsas de plástico. La preparación del sedimento experimental consistió en el secado en estufa a $80^{\circ}$ C durante 24 h (To-orn \& Paphavasit, 2017) y el tamizado a través de una malla de $250 \mu \mathrm{m}$ para utilizar la parte menor de esta fracción en el ensayo (Méndez, Lacorte, \& Barata, 2013).

Diseño experimental: Para el ensayo de mortalidad se utilizaron únicamente adultos de Capitella sp., debido a que su mayor tamaño facilita la manipulación en el laboratorio. Los machos presentan espinas genitales entre el octavo y noveno setígero torácico y las hembras se distinguen por la presencia de ovarios amarillos, ovocitos blancos o incluso dentro de tubos incubadores (Méndez, 2016). Durante el ensayo se monitorearon los valores de oxígeno disuelto, temperatura y salinidad; sin embargo, para el diseño experimental se consideraron únicamente las dos variables ambientales debido a que ambos parámetros se pueden controlar con mayor facilidad en el laboratorio, mientras que el oxígeno disuelto puede variar al introducir alimento a los acuarios. Se realizaron dos tratamientos con diferentes intervalos de temperatura y salinidad del agua:

Tratamiento 1. Se utilizaron los especímenes de Capitella sp. recolectados durante enero de 2017 y los intervalos de temperatura $\left(20-22^{\circ} \mathrm{C}\right)$ y salinidad (30-32 ups) fueron determinados de acuerdo con aproximaciones de los valores de temperatura y salinidad medidos en el campo durante esa época del año.

Tratamiento 2. Se utilizaron los individuos de Capitella sp. recolectados durante noviembre de 2016. En este caso, los intervalos de temperatura y salinidad fueron determinados tomando en cuenta los datos registrados por varios autores durante sus experiencias en el laboratorio con varias especies del complejo de Capitella spp. procedentes de distintas localidades del mundo, tales como Forbes, Forbes, \& Holmer (1996); Gamenick et al. (1998); 
Linke-Gamenick et al. (2000); Méndez (2002); Méndez (2005); Méndez \& GreenRuiz (2005); Méndez (2006a); Méndez (2006b); Méndez et al. (2008); Méndez et al. (2013). Estos valores se ha observado que favorecen la supervivencia de Capitella spp. en el laboratorio. Así, los intervalos para este tratamiento fueron de 24 a $26^{\circ} \mathrm{C}$ y de 32 a 34 ups. Se intentó mantener la temperatura del agua dentro de los intervalos indicados en diferentes mesas de enfriamiento, mientras que los intervalos de salinidad se prepararon, inicialmente, mediante la adición de agua destilada al agua de mar, ya que se utilizó agua de mar de las cisternas para el cultivo de conchas de abanico que tenía una salinidad de 35 ups.

El ensayo de mortalidad bajo condiciones de laboratorio se realizó según los criterios de Horng et al. (2009). Para cada tratamiento se realizaron cinco réplicas, cada una en un vaso de precipitados de $250 \mathrm{~mL}$. Cada réplica consistió en $2-4 \mathrm{~cm}$ de sedimento experimental, $250 \mathrm{~mL}$ de agua de mar filtrada $(<1 \mu \mathrm{m})$ y 20 individuos completos de Capitella sp. El ensayo fue monitoreado durante nueve días midiendo las variables ambientales (salinidad, temperatura del agua y oxígeno disuelto). La alimentación de los especímenes se realizó mediante la adición, cada tres días, de $0.1 \mathrm{~g}$ de alimento artificial que consistió en la mezcla en partes iguales de comida para peces (Tetramin) y espinaca seca tamizada a menos de $250 \mu \mathrm{m}$ (Moore \& Dillon, 1993; Forbes et al., 1996; Méndez, 2015).

Análisis de datos: Los especímenes vivos fueron contados diariamente utilizando el microscopio estereoscópico, a fin de determinar su supervivencia. El porcentaje de supervivencia para cada réplica se determinó de acuerdo con la expresión propuesta por Horng et al. (2009): $\mathrm{S}=(\mathrm{nf} / \mathrm{ni}) * 100$, donde $\mathrm{S}=\%$ de supervivencia, $\mathrm{nf}=$ número final de individuos vivos, $\mathrm{ni}=$ número inicial de individuos vivos . Los datos de supervivencia fueron expresados como porcentaje de mortalidad por día en cada una de las réplicas.

La prueba de Kolmogorov-Smirnov demostró que los datos no son normales, por lo que se realizaron análisis estadísticos no paramétricos. A fin de determinar si existe correlación entre la mortalidad con cada una de las variables ambientales, se utilizó el índice de Correlación de Spearman. Los resultados fueron considerados como significativos cuando la probabilidad de cometer error fue menor a 0.05. Los análisis se realizaron utilizando el programa Statistica (versión 10).

Para conocer si hay diferencias entre los tratamientos, se realizó la prueba de Kolmogorov-Smirnov para dos muestras. El procedimiento consistió en obtener las frecuencias relativas acumuladas del número de individuos muertos y la proporción (valor observado), obtenido de la frecuencia acumulada y dividida entre el total de la muestra, por día para cada tratamiento, con el fin de hallar la diferencia máxima entre los tratamientos. El valor obtenido se comparó con el valor tabular (valor teórico) de K-S.

Valor tabular de K-S:

$$
\mathrm{D} \text { tab. }=1.36 \sqrt{\frac{n_{1}+n_{2}}{n_{1} \times n_{2}}}
$$

$n_{1}$ : total de individuos del tratamiento 1

$n_{2}$ : total de individuos del tratamiento 2

Se cumple:

D observado $<$ D $\alpha$ tabular: $\mathrm{H}_{0}=$ no hay diferencias entre ambos tratamientos

D observado $>$ D $\alpha$ tabular: $\mathrm{H}_{\mathrm{a}}=$ hay diferencias entre ambos tratamientos

Para determinar si hay variación en la mortalidad dentro de cada tratamiento, entre sus cinco réplicas por día, se calculó el coeficiente de variación (C.V):

$$
\mathrm{C} . \mathrm{V}=\frac{s * 100 \%}{x}
$$

Donde:

$s$ : desviación estándar

$\bar{x}$ : promedio aritmético 


\section{RESULTADOS}

Los valores de temperatura, salinidad y oxígeno disuelto medidos en el agua durante el ensayo se compilan en la tabla 1. En ambos tratamientos se observaron valores que están fuera de los intervalos de temperatura y salinidad previamente establecidos, lo que produjo una evidente ampliación de dichos intervalos, calculados como la diferencia entre los valores máximos y mínimos (tratamiento 1: $8^{\circ} \mathrm{C}$ y 12 ups; tratamiento $2: 8.3^{\circ} \mathrm{C}$ y 11.4 ups). Asimismo, el oxígeno disuelto presentó grandes oscilaciones en ambos tratamientos (tratamiento 1: 0.004-67.48 $\mathrm{mL} / \mathrm{L}$; tratamiento 2: $0.08-8.8 \mathrm{~mL} / \mathrm{L})$.

El índice de correlación de Spearman mostró que, en ambos tratamientos, la mortalidad presentó correlación positiva únicamente con la temperatura y con el tiempo, mientras que la salinidad y el oxígeno disuelto no mostraron correlación con la mortalidad (Tabla 2). Los porcentajes de mortalidad al final del ensayo en las cinco réplicas fueron $35 \%, 40 \%, 95 \%, 55$

TABLA 1

Temperatura del agua, salinidad y oxígeno disuelto registrados durante el ensayo

TABLE 1

Water temperature, salinity and dissolved oxygen recorded during the bioassay

\begin{tabular}{|c|c|c|c|c|c|c|c|}
\hline \multirow[b]{2}{*}{ Réplicas } & \multirow{2}{*}{$\begin{array}{c}\text { Tiempo } \\
\text { (días) }\end{array}$} & \multicolumn{3}{|c|}{ Tratamiento 1} & \multicolumn{3}{|c|}{ Tratamiento 2} \\
\hline & & $\begin{array}{c}\text { Temperatura } \\
\left({ }^{\circ} \mathrm{C}\right)\end{array}$ & $\begin{array}{l}\text { Salinidad } \\
\text { (ups) }\end{array}$ & $\begin{array}{l}\text { Oxígeno } \\
(\mathrm{ml} / \mathrm{l})\end{array}$ & $\begin{array}{c}\text { Temperatura } \\
\left({ }^{\circ} \mathrm{C}\right)\end{array}$ & $\begin{array}{l}\text { Salinidad } \\
\text { (ups) }\end{array}$ & $\begin{array}{c}\text { Oxígeno } \\
(\mathrm{ml} / \mathrm{l})\end{array}$ \\
\hline \multirow[t]{9}{*}{1} & 1 & 21.60 & 29.4 & 2.79 & 23 & 31.70 & 3.82 \\
\hline & 2 & 22 & 34.1 & 0.45 & 24.9 & 33.40 & 0.08 \\
\hline & 3 & 22.80 & 31.1 & 2.3 & 25.5 & 34.70 & 6.14 \\
\hline & 4 & 29.60 & 35.9 & 2.44 & 24.4 & 35.30 & 2.37 \\
\hline & 5 & 22.60 & 34.3 & 5.29 & 24.2 & 31.40 & 5.82 \\
\hline & 6 & 22.50 & 31.8 & 4.9 & 22.9 & 30.10 & 5.19 \\
\hline & 7 & 22.90 & 32 & 5.99 & 24.5 & 27.10 & 7.01 \\
\hline & 8 & 23.50 & 32 & 0.33 & 23.8 & 28.90 & 3.74 \\
\hline & 9 & 23.10 & 33 & 2.3 & 24.3 & 29.90 & 8.80 \\
\hline \multirow[t]{9}{*}{2} & 1 & 21.6 & 32.4 & 7.48 & 22.9 & 32.6 & 3.76 \\
\hline & 2 & 21.9 & 32.7 & 0.03 & 25.2 & 34.3 & 0.08 \\
\hline & 3 & 21.8 & 31.6 & 3.15 & 24.4 & 30.9 & 0.11 \\
\hline & 4 & 22.4 & 36.6 & 0.22 & 25.9 & 32.4 & 0.17 \\
\hline & 5 & 23.1 & 31.7 & 0.96 & 25.2 & 33.9 & 7.43 \\
\hline & 6 & 22.4 & 32 & 6.17 & 24.2 & 34.4 & 7.57 \\
\hline & 7 & 23 & 29.6 & 6.8 & 25.3 & 35.6 & 1.76 \\
\hline & 8 & 23.6 & 31.8 & 5.02 & 24 & 35.2 & 4.05 \\
\hline & 9 & 22.7 & 33.1 & 1.53 & 23.1 & 35.5 & 6.69 \\
\hline \multirow[t]{9}{*}{3} & 1 & 21.60 & 31.3 & 3.76 & 22.9 & 29 & 2.85 \\
\hline & 2 & 21.90 & 31.6 & 0.13 & 25.9 & 31.7 & 0.37 \\
\hline & 3 & 21.90 & 30.6 & 0.004 & 22.3 & 33.1 & 0.08 \\
\hline & 4 & 23.10 & 36.7 & 0.14 & 35 & 24.4 & 4.67 \\
\hline & 5 & 22.70 & 33.3 & 6.34 & 24.9 & 35.7 & 2.49 \\
\hline & 6 & 22.70 & 33.3 & 0.06 & 25.4 & 32.5 & 5.38 \\
\hline & 7 & 23.00 & 30.6 & 6.6 & 24.7 & 32.6 & 5.77 \\
\hline & 8 & 23.50 & 30.4 & 5.9 & 24.3 & 33.4 & 0.12 \\
\hline & 9 & 22.60 & 32.6 & 4.17 & 23.8 & 33.7 & 7.28 \\
\hline 4 & 1 & 21.70 & 31.9 & 4.7 & 22.9 & 29 & 2.85 \\
\hline
\end{tabular}


Tabla 1 (Continuación) / TABLE 1 (Continued)

\begin{tabular}{|c|c|c|c|c|c|c|c|}
\hline \multirow[b]{2}{*}{ Réplicas } & \multirow[b]{2}{*}{$\begin{array}{c}\text { Tiempo } \\
\text { (días) }\end{array}$} & \multicolumn{3}{|c|}{ Tratamiento 1} & \multicolumn{3}{|c|}{ Tratamiento 2} \\
\hline & & $\begin{array}{c}\text { Temperatura } \\
\left({ }^{\circ} \mathrm{C}\right)\end{array}$ & $\begin{array}{l}\text { Salinidad } \\
\text { (ups) }\end{array}$ & $\begin{array}{l}\text { Oxígeno } \\
(\mathrm{ml} / \mathrm{l})\end{array}$ & $\begin{array}{c}\text { Temperatura } \\
\left({ }^{\circ} \mathrm{C}\right)\end{array}$ & $\begin{array}{l}\text { Salinidad } \\
\text { (ups) }\end{array}$ & $\begin{array}{c}\text { Oxígeno } \\
(\mathrm{ml} / \mathrm{l})\end{array}$ \\
\hline \multirow{17}{*}{5} & 2 & 21.90 & 32.1 & 0.44 & 25.4 & 30.9 & 5.5 \\
\hline & 3 & 22.50 & 31.5 & 0.46 & 24.9 & 31.9 & 4.56 \\
\hline & 4 & 22.30 & 29.4 & 6.34 & 23 & 31.6 & 5.2 \\
\hline & 5 & 22.50 & 31.5 & 5.06 & 25.6 & 31.6 & 7.4 \\
\hline & 6 & 22.70 & 34.3 & 0.24 & 23.8 & 31.9 & 4.17 \\
\hline & 7 & 23.00 & 32.2 & 4.97 & 25 & 31.9 & 6.79 \\
\hline & 8 & 23.00 & 32.1 & 1.13 & 24.9 & 32.1 & 0.18 \\
\hline & 9 & 23.00 & 32.30 & 1.13 & 24 & 32.4 & 6.95 \\
\hline & 1 & 21.7 & 29.3 & 2.43 & 23.9 & 34.3 & 7.39 \\
\hline & 2 & 21.9 & 35.1 & 4.32 & 24.1 & 35.8 & 7.5 \\
\hline & 3 & 21.8 & 30.8 & 0.18 & 24 & 34.4 & 7.07 \\
\hline & 4 & 22.5 & 37.6 & 6.45 & 23.9 & 35.4 & 7.97 \\
\hline & 5 & 22.7 & 30 & 0.15 & 21.7 & 34.6 & 2.43 \\
\hline & 6 & 22.5 & 33.3 & 0.06 & 22.5 & 33.3 & 3.3 \\
\hline & 7 & 23 & 33.4 & 0.95 & 22.5 & 31.8 & 4.9 \\
\hline & 8 & 23.1 & 31.9 & 0.25 & 23.1 & 31.9 & 0.25 \\
\hline & 9 & 22.6 & 32.2 & 1.43 & 22.6 & 32.2 & 2.05 \\
\hline
\end{tabular}

TABLA 2

Índice de correlación de Spearman entre la mortalidad y las variables ambientales y el tiempo (en negritas, valores significativos a $\mathrm{p}<0.05)$.

TABLE 2

Spearman correlation index among mortality and the environmental variables and time (in bold letters, significant values at $\mathrm{p}<0.05)$.

\begin{tabular}{lc} 
& Mortalidad \\
TRATAMIENTO 1 & \\
Mortalidad & 1.000 \\
Tiempo & 0.790 \\
Temperatura & 0.631 \\
Oxígeno & 0.100 \\
Salinidad & 0.034 \\
& \\
TRATAMIENTO 2 & \\
Mortalidad & 1.000 \\
Tiempo & 0.915 \\
Temperatura & 0.705 \\
Oxígeno & 0.063 \\
Salinidad & 0.076 \\
\hline
\end{tabular}

$\%$ y $75 \%$ para el tratamiento 1 (enero) y $65 \%$, $95 \%, 85 \%, 60 \%, 65 \%$ para el tratamiento 2 (noviembre). La figura 1 muestra el incremento de la mortalidad a lo largo del tiempo, la cual, fue mayor en el tratamiento $2(74 \%)$ que en el tratamiento $1(60 \%)$.

No se observaron diferencias entre los tratamientos por la prueba de K-S. Sin embargo, se observó variabilidad porcentual en el coeficiente de variación entre los días por tratamiento. En noviembre se observó el incremento de la variabilidad conforme los días transcurrían, contrario a lo observado en el mes de enero, donde a partir de la mitad del experimento se observó estabilidad (Tabla 3).

\section{DISCUSIÓN}

Los resultados de este estudio mostraron que la mortalidad de los especímenes de Capitella $\mathrm{sp}$. estuvo influenciada por el incremento en la temperatura y por el paso del tiempo, 

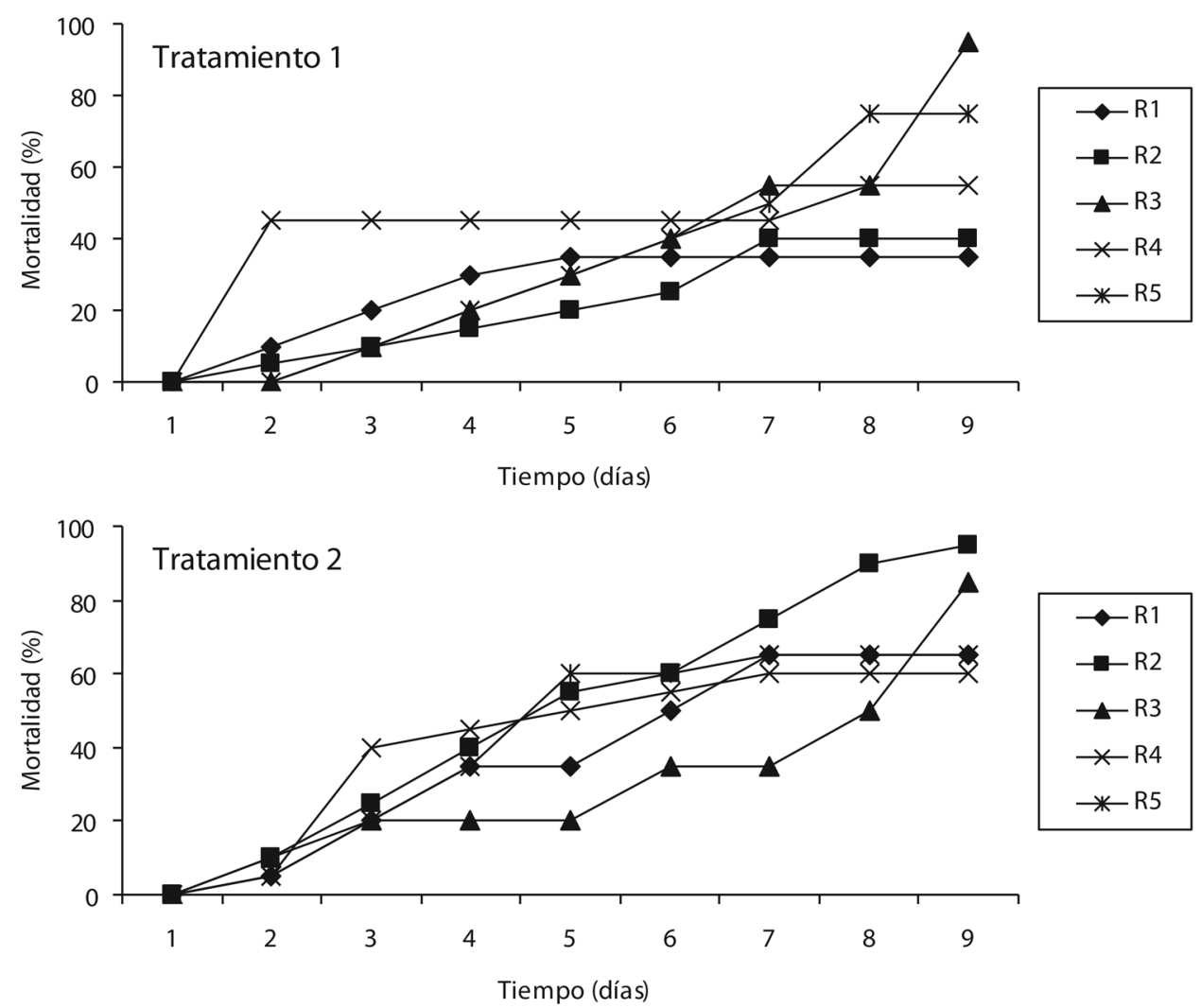

Fig. 1. Mortalidad (\%) de adultos de Capitella sp. registrada en los dos tratamientos durante el ensayo (Tratamiento 1: 20-22 ${ }^{\circ} \mathrm{C}$ y $30-32$ ups; tratamiento $2: 24-26^{\circ} \mathrm{C}$ y 32 a 34 ups); (R= réplicas).

Fig. 1. Mortality (\%) of adults of Capitella sp. recorded in the treatments during the bioassay (Treatments $1: 20-22^{\circ} \mathrm{C}$ y 30-32 ups; treatments $2: 24-26^{\circ} \mathrm{C}$ y 32 a 34 ups); ( $\mathrm{R}=$ replicates).

de manera que los mayores porcentajes de mortalidad se registraron en la segunda mitad de cada tratamiento (Fig. 1). La diferencia en mortalidad entre los dos tratamientos también puede ser explicada en términos de aclimatación a las condiciones de laboratorio. El hecho de presentar intervalos de temperatura y salinidad cercanas a los de su hábitat, pudo producir que los organismos de enero (tratamiento 1) estuvieran mejor aclimatados a las condiciones de laboratorio durante el experimento, lo que les permitió tener una mayor supervivencia que los organismos de noviembre (tratamiento 2) donde el cambio fue más drástico. La mayor supervivencia de los organismos recolectados en enero puede ser resultado de que la mayoría de los intervalos de temperatura y salinidad a los que fueron sometidos durante el ensayo correspondían a los registrados en el medio natural durante su recolección. Este comportamiento también se evidencia en el coeficiente de variación, donde los valores de temperatura, se estabilizaron a mitad del primer tratamiento, sugiriendo que los intervalos de temperatura de su hábitat serían los óptimos para su cultivo en el laboratorio.

Esto se puede explicar porque la temperatura es un factor importante que determina los parámetros biológicos en la comunidad bentónica dentro del ecosistema marino al estar vinculada a los procesos físicos-químicos del sedimento y columna de agua, influyendo en su distribución, abundancia y flujo energético (Hutching, 1998; Méndez, 2007; Liu 
TABLA 3

Variación porcentual del coeficiente de variación del tratamiento 1 (enero 2017) y tratamiento 2 (noviembre 2016) de la mortandad de las cinco réplicas de los nueve días del bioensayo

TABLE 3

Percent variation of the coefficient of variation of treatments 1 (January 2017) and treatments 2

(November 2016) of the mortality of the five replicates of the nine days of the bioassay

\begin{tabular}{ccc}
$\mathrm{N}^{\circ}$ días & $\begin{array}{c}\text { Noviembre } \\
\text { Temperatura:24-26 }{ }^{\circ} \mathrm{C} \\
\text { Salinidad: } 32-34 \text { ups. }\end{array}$ & $\begin{array}{c}\text { Enero } \\
\text { Temperatura: } 20-22^{\circ} \mathrm{C} \\
\text { Salinidad: } 30-32 \text { ups }\end{array}$ \\
\hline 1 & 0.00 & 0.00 \\
2 & 39.12 & 127.28 \\
3 & 54.15 & 63.89 \\
4 & 70.71 & 63.89 \\
5 & 108.33 & 69.72 \\
6 & 50.00 & 108.65 \\
7 & 83.85 & 141.42 \\
8 & 136.93 & 115.47 \\
9 & 190.60 & 0.00 \\
\hline
\end{tabular}

\& Xian, 2009; Custodio \& Cosme, 2016; Jiménez, 2016).

Cuando los valores de temperatura, salinidad, $\mathrm{pH}$ no son óptimos puede generar estrés oxidativo en el organismo (Pires, Figueira, Moreira, Soares, \& Freitas, 2015), como por ejemplo, el aumento de temperatura que ocasiona la disminución de la disponibilidad de oxígeno dentro del ecosistema (GutiérrezGaraviz, Zamora, \& Andrade-Sossa, 2014; Hahn-vonHessberg, Toro, Grajales-Quíntero, Duque-Quíntero, Serna-Uribe, 2009; Levin 2003; Levin et al., 2009), originando que los organismos utilicen diferentes mecanismos fisiológicos para adaptarse a nuevas condiciones ambientales, situación que se observó en el tratamiento 2 donde se hubo mayor variabilidad.

El cambio de temperatura también influye en los requerimientos metabólicos y procesos relacionados con la transformación y el consumo del alimento, en el crecimiento (Prevedelli, 1991; Calderer, 2001), equilibrio osmótico y función enzimática (Snelgrove, 1999), flujo de energía y procesos fisiológicos (Liu \& Xian,
2009). Por ejemplo, Liu \& Xian (2009) señalan que temperaturas anormales ocasionarían el incremento de la excreción del nitrógeno como resultado del incremento catabólico en los aminoácidos o en la síntesis de péptidos, relacionados a la regulación osmótica. La temperatura es también un factor influyente en la fecundidad durante la etapa reproductiva, en el tamaño de los huevos, y en el desarrollo de las fases larvales y juveniles en los poliquetos (Quian \& Chia, 1992).

La reproducción de las condiciones del hábitat en el laboratorio ha sido recomendada para cultivar las especies del complejo Capitella (Méndez \& Green-Ruiz, 2006). Asimismo, Barria, Jara, \& Paschke (2005), en su estudio de laboratorio sobre la temperatura como factor de variabilidad en el desarrollo y morfología de larvas zoea del braquiuro Acanthocyclus hassleri, mantuvieron a las hembras ovígeras bajo condiciones de temperatura y salinidad similares a las de su hábitat.

Varios estudios ecotoxicológicos y de reproducción de Capitella spp. desarrollados en el laboratorio (mencionados en Méndez, 2015) se caracterizan por el control de la temperatura y salinidad para evitar posibles cambios fisiológicos en los organismos producidos por estas variables o que actúen sinérgicamente con otros factores ambientales o con sustancias tóxicas. Este hecho demuestra la importancia de estas variables, a pesar de que la salinidad no presentó un efecto significativo en la mortalidad de Capitella sp. La ausencia de efecto significativo de la salinidad y el oxígeno en la mortalidad de Capitella no necesariamente indica que estas dos variables no afecten el desarrollo de la especie; pudo existir un efecto sinérgico de las tres variables analizadas sobre la mortalidad de algunos organismos de cada tratamiento, como lo demostró Kenny (1969) con los poliquetos Diopatra cuprea y Clymenella torquata. Esto no es de extrañar, debido a la fuerte correlación que, de manera natural, existe entre las tres variables ambientales, como lo indican Silva \& Guerra (2008).

La purificación del agua para nuestro ensayo se realizó mediante una bomba al vacío 
utilizando un papel filtro de $1 \mu \mathrm{m}$. Para la producción intensiva del poliqueto Nereis diversicolor, García del Real, Canteras, \& Valcarce (2007) utilizaron sistemas de depuración que consistían en filtros de grava, arena y conchas y un catalizador de urea, que permitían una calidad de agua ideal con niveles de amonio, nitritos y nitratos adecuados para el cultivo de esta especie. Asimismo, Mohammad (2015) hace referencia a un sistema de recirculación en acuacultura que permite diferentes procesos de filtración tanto mecánica como biológica, utilizando agentes físicos como oxígeno, temperatura, ozono, UV, $\mathrm{pH}$ y salinidad para la remoción de sustancias residuales. Este sistema resultó muy efectivo en el cultivo de $N$. diversicolor a base de diferentes dietas que permitieron el $99.3 \%$ y $95.3 \%$ de supervivencia. A pesar de que los miembros de Capitella spp. son tolerantes a condiciones degradadas, la mortalidad registrada pudo reducirse mejorando la calidad del agua de mar utilizada durante el experimento, empleando los métodos indicados previamente.

La mortalidad observada hacia la segunda parte del experimento puede atribuirse, además, a varios factores no controlados durante el experimento, tales como la edad, el estado de salud y estado de madurez de los organismos, que pudieron morir de manera natural a lo largo del tiempo. Asimismo, la adición de alimento artificial pudo alterar la cantidad de materia orgánica en el sedimento experimental y, por lo tanto, el contenido de oxígeno disuelto, el cual osciló entre 0.004 y $7.48 \mathrm{~mL} / \mathrm{L}$ en el tratamiento 1 y entre 0.08 y $8.8 \mathrm{~mL} / \mathrm{L}$ en el tratamiento 2 (Tabla 1). Si bien los capitélidos se caracterizan por tolerar condiciones hipóxicas en los sedimentos (Diaz \& Rosenberg, 1995; Forbes et al, 1996; Gamenick et al., 1998; Linke-Gamenick et al., 2000a), estos cambios repentinos pudieron descompensar a algunos individuos provocándoles la muerte.

La presente investigación es el primer esfuerzo en Perú para conocer las mejores condiciones para el mantenimiento y reproducción de Capitella spp. en el laboratorio, con el fin de realizar futuros estudios ecológicos, ecotoxicológicos, biotecnológicos y de reproducción. Para ello, se sugiere la realización de estudios a largo plazo utilizando equipos potentes para las mediciones y mantenimiento de los parámetros fisicoquímicos, especialmente la temperatura. Se recomienda intentar reproducir las condiciones ambientales del hábitat donde viven los organismos, con el fin de mantener el cultivo de una población bajo condiciones óptimas y alcanzar porcentajes de mortalidad cercanos al $0 \%$.

Declaración de ética: los autores declaran que todos están de acuerdo con esta publicación y que han hecho aportes que justifican su autoría; que no hay conflicto de interés de ningún tipo; y que han cumplido con todos los requisitos y procedimientos éticos y legales pertinentes. Todas las fuentes de financiamiento se detallan plena y claramente en la sección de agradecimientos. El respectivo documento legal firmado se encuentra en los archivos de la revista.

\section{AGRADECIMIENTOS}

Agradecemos a Christian Paredes, jefe del Laboratorio de Ecotoxicología Acuática del Instituto del Mar del Perú (IMARPE), por sus comentarios y correcciones, así como por el apoyo brindado durante la estancia de investigación de MHP. Igualmente, a Sofía Reyes Grimaldo, Hayde López Cabanillas, Ricardo Dioses Avellana, Manuel Mendoza Antón y Aida Henostroza Quiroz por su apoyo y sugerencias.

\section{RESUMEN}

Introducción: El poliqueto marino y estuarino Capitella capitata es una especie endobentónica y considerado cosmopolita. El cultivo en el laboratorio de este complejo de especies permite su utilización como especie para pruebas en bioensayos de toxicidad, así como para estudios de reproducción. Objetivo: El presente trabajo pretende observar la mortalidad en dos rangos de temperatura y salinidad como condiciones óptimas para cultivar Capitella sp. en el laboratorio. Metodología: La recolección de especímenes se realizó en la provincia de Callao, Perú en 
noviembre de 2016 y enero de 2017. Se realizó un bioensayo para observar la mortalidad bajo diferentes intervalos de temperatura y salinidad: tratamiento 1 con los organismos recolectados en enero y con intervalos de temperatura (20$22^{\circ} \mathrm{C}$ ) y salinidad (30-32 ups) similares a los registrados en el campo; tratamiento 2, con los organismos recolectados en noviembre y con intervalos de temperatura $\left(24-26{ }^{\circ} \mathrm{C}\right)$ y salinidad (32 a 34 ups) seleccionados de acuerdo con datos de la bibliografía. Resultados: El aumento de la temperatura y el tiempo influyeron significativamente en la mortalidad de los organismos de ambos tratamientos (Correlación de Spearman), mientras que la salinidad y el oxígeno no presentaron correlación significativa con la mortalidad. Se observó efecto significativo del tiempo en la mortalidad, con más muertes a partir de la segunda mitad del experimento. Los organismos del tratamiento 1, con condiciones ambientales similares a las de su hábitat sobrevivieron más que los del tratamiento 2. Estos resultados también se observaron en la variabilidad porcentual en el coeficiente de variación entre los días por tratamiento. No se observaron diferencias entre los tratamientos al comparar las curvas acumuladas de mortalidad. Conclusión: Los intervalos óptimos para el cultivo en el laboratorio de Capitella sp. son los de su hábitat. Las posibles causas de la mortalidad observada se atribuyen, además de la temperatura y el tiempo, a la edad, estado de salud o de madurez de los organismos y, probablemente, la mala calidad del agua utilizada en el bioensayo.

Palabras clave: Poliquetos, supervivencia, bioensayo, temperatura, salinidad, oxígeno disuelto.

\section{REFERENCIAS}

Aguirre, L. \& Canales, R. (2017). Poliquetos de Perú: estado actual y perspectivas para la investigación. En O. Díaz-Díaz, D. Bone, C. T. Rodríguez, \& V. H. Delgado-Blas (Eds.) Poliquetos de Sudamérica (pp. 101-114). Cumaná, Venezuela: Volumen especial del Boletín del Instituto Oceanográfico de Venezuela.

Barria, E. M., Jara, C. G., \& Paschke, K. A. (2005). La temperatura como factor de variabilidad en el desarrollo y morfología de larvas zoeas de Acanthocyclus hassleri Rathbun (Decapoda, Brachyura, Atelecyclidae) cultivadas en el laboratorio. Investigaciones Marinas, Valparaíso. 33, 25-41.

Blake, J. A. (2009). Redescription of Capitella capitata (Fabricius) from West Greenland and designation of a neotype (Polychaeta, Capitellidae), Zoosymposia, 2, 55-80

Blake, J. A., Grassle, J. P., \& Eckelbarger, K. J. (2009). Capitella teleta, a new species designation for the opportunistic and experimental Capitella sp. I, with a review of the literature for confirmed records. Zoosymposia, 2, 25-53.
Calderer, R. A. (2001). Influencia de la Temperatura y la Salinidad sobre el crecimiento y consumo de oxígeno de la Dorada (Sparus aurata L.) (Tesis de doctorado). Barcelona, España: Universidad de Barcelona.

Custodio V. M., \& Cosme C. Z. F. (2016). Análisis de la biodiversidad de macroinvertebrados bentónicos del río Cunas mediante indicadores ambientales, JunínPerú. Scientia Agropecuaria, 7(19), 33-44.

Dean, H. K. (2008). The use of polychaetes (Annelida) as indicator species of marine pollution: a review. Revista de. Biología Tropical, 56(4), 11-38.

Diaz, R. J., \& Rosenberg, R. (1995). Marine benthic hypoxia: a review of its ecological effects and the behavioural responses of benthic macrofauna. Oceanography and Marine Biology, An Annual Review, 33, 245-303.

Eckelbarger, K. J., Linley, P. A., \& Grassle, J. P. (1984). Role of ovarian follicle cells in vitellogenesis and oocyte resorption in Capitella sp. I. (Polychaeta). Marine Biology, 79, 133-144.

Eckelbarger, K. J., \& Grassle, J. P. (1987). Spermatogenesis, sperm storage and comparative sperm morphology in nine species of Capitella, Capitomastus and Capitellides (Polychaeta: Capitellidae). Marine Biology, 95, 415-429.

Fauchald, K. (1977). The polychaete worms. Definitions and keys to the orders, families and genera. Natural History Museum of Los Angeles County, and Allan Hancock Foundation. Science Series, 8, 1-188.

Fidalgo e Costa, P., Passos, A. M., \& Cancela da Fonseca, L. (2003). Polychaetes and their potential use in aquaculture. World Aquaculture, 34(3), 41-43.

Forbes, V., Forbes, T., \& Holmer, M. (1996). Inducible metabolism of fluoranthene by the opportunistic polychaete Capitella sp. I. Marine Ecology Progress Series, 132, 63-70.

Gambi, M. C., Castelli, A., Giangrande, A., Lanera, P., Prevedelli, D., Vandini, R. Z. (1994). Polychaetes of commercial and applied interest in Italy: an overview. En J. C. Dauvin, L. Laubier, D. J. Reish, (Eds.). Actes de la 4 e me Confrence Internationale des Polychètes (pp 593-602, Vol. 162). Paris, Francia: Memoires du Musum National d' Histoire Naturelle.

Gamenick, I, Vismann, B., \& Grieshaber, M. K. (1998). Ecophysiological differentiation of Capitella capitata (Polychaeta). Sibling species from different sulfidic habitats. Marine Ecology Progress Series, $175,155-166$.

García del Real, J. M., Canteras, J. C., \& Valcarce, A. (2007). Proyecto para el desarrollo de técnicas aplicables al cultivo de gusanos marinos para su producción intensiva. Locustella. Anuario de la Naturaleza de Cantabria, 4, 66-75. 
Grémare, A., Marsh, A. G., \& Tenore, K. R. (1989). Fecundity and energy partitioning in Capitella capitata type I (Annelida: Polychaeta). Marine Biology, 100, 365-371.

Gutiérrez-Garaviz, J., Zamora, G. H., \& Andrade-Sossa, C. E. (2014). Efecto de la actividad antrópica sobre la composición y diversidad de macroinvertebrados acuáticos en el río Cofre (Sistema lótico andino colombiano). Revista Biodiversidad Neotropical, 4(2), 113-23.

Hahn-vonHessberg, C.M., Toro, D.R., Grajales-Quíntero, A., Duque-Quíntero, G., \& Serna-Uribe, L. (2009). Determinación de la Calidad del Agua mediante indicadores biológicos y fisicoquímicos, en la estación piscícola, Universidad de Caldas, Municipio de Palestina, Colombia. Boletín Científico Centro de Museos Museo de Historia Natural, 13(2), 89-105.

Halanych, K. M., \& Borda, E. (2009). Developing models for Lophotrochozoan and Annelid Biology. En D. H. Shain (Ed.) Annelids in Modern Biology (pp. 3-12). New Jersey, USA: Wiley-Blackwell.

Horng, C. Y., Wang, S. L, \& Cheng, I. J. (2009). Effects of sediment-bound $\mathrm{Cd}, \mathrm{Pb}$, and $\mathrm{Ni}$ on the growth, feeding, and survival of Capitella sp. I. Journal of Experimental Marine Biology and Ecology, 371, 68-76.

Hutching P. (1998). Biodiversity and functioning of polychaetes in benthic sediments. Biodiversity and Conservation, 7, 1133-1145.

Jiménez C. A. (2016). Asentamiento y reclutamiento de poliquetos bentónicos en la plataforma continental frente a Callao desde verano a invierno de 2015 (Tesis de maestría). Lima, Perú: Universidad Peruana Cayetano Heredia.

Kenny, R. (1969). Temperature tolerance of the polychaete worms Diopatra cuprea and Clymenella torquata. Marine Biology, 4, 219-223.

Levin, L. A. (2003). Oxygen minimum zone benthos: adaptation and community response to hypoxia. Oceanography and Marine Biology: An Annual Review. $41,1-45$.

Levin, L. A., Ekau, W., Gooday, A. J., Jorissen, F., Middelburg, J. J., Naqvi, S. W. A., ... Zhang, J. (2009). Effects of natural and human-induced hypoxia on coastal benthos. Biogeosciences, 6(10), 2063-2098.

Linke-Gamenick, I., Vismann, B., \& Forbes, V. E. (2000a). Effects of fluoranthene and ambient oxygen levels on survival and metabolism in three sibling species of Capitella (Polychaeta). Marine Ecology Progress Series, 194, 169-177.

Linke-Gamenick, I., Forbes, V. E., \& Méndez, N. (2000b). Effects of chronic fluoranthene exposure on sibling species of Capitella with different development modes. Marine Ecology Progress Series, 203, 191-203.

Liu, Y., \& Xian W. (2009). The effect of temperatura on Growth and Energy Budget of the Polychaete, Neanthes japónica Izuka. Oceanic and Coastal Sea Research, 8(2), 177-183.

Méndez, N. (1995). Non-pelagic development of Capitella capitata (Polychaeta) in Barcelona, Spain. Scientia Marina, 59, 95-101.

Méndez, N. (2002). Experimental evidence of polymophysm of sexual development in Capitella sp B (Polychaeta: Capitellidae) from Barcelona, Spain. Scientia Marina, 66(2), 103-110.

Méndez, N. (2005). Effects of teflubenzuron on larvae and juveniles of the polychaete Capitella sp B from Barcelona, Spain. Water, Air, and Soil Pollution, 160, 259-269.

Méndez, N., \& Green-Ruiz, C. (2005). Preliminary observations of cadmium and copper effects on juveniles of the polychaete Capitella sp. Y (Annelida: Polychaeta) from Estero del Yugo, Mazatlán, México. Revista Chilena de Historia Natural, 78, 701-710.

Méndez, N. (2006a). Life cycle of Capitella sp Y (Polychaeta: Capitellidae) from Estero del Yugo, Mazatlan, Mexico. Journal of the Marine Biological Association of the United Kingdom, 86, 263-269.

Méndez, N. (2006b). Effects of teflubenzuron on sediment processing by members of the Capitella speciescomplex. Environmental Pollution, 139, 118-124.

Méndez, N., \& Green-Ruiz, C. (2006). Advantages and disadvantages of performing ecotoxicological bioassays with larvae of polychaetes belonging to the Capitella capitata species-complex. Oceánides, 21(1, 2), 145-151.

Méndez, N. (2007). Relationships between deep-water polychaete fauna and environmental factors in the southeastern Gulf of California, Mexico. Scientia Marina 71, 605-622.

Méndez, N., Anguas-Cabrera, D. N., \& García-de la Parra, L. M. (2008). Effect of methamidophos on sediment processing and body mass of Capitella sp Y from Estero del Yugo, Mazatlan, Mexico. Journal of Experimental Marine Biology and Ecology, 361, 92-97.

Méndez, N., Lacorte, S., \& Barata, C. (2013). Effects of the pharmaceutical fluoxetine in spiked-sediments on feeding activity and growth of the polychaete Capitella teleta. Marine Environmental Research, 89, 76-82.

Méndez, N. (2015). El poliqueto Capitella spp. como especie prueba en estudios de ecotoxicología. Heosphoros: Revista de Investigación Multidisciplinaria, $1,46-54$. 
Méndez, N., \& Barata, C. (2015). Effects of the antidepressant fluoxetine in spiked-sediments on developmental and reproductive features of the polychaetes Capitella teleta and Capitella sp A. Ecotoxicology, 24, 106-118.

Méndez, N. (2016). Laboratory development of Capitella sp. A (Annelida: Capitellidae) from a NW Mediterranean fish farm reared under different organic enrichment conditions. Scientia Marina, 80(4), 535-542.

Mohammad, K. M. L. (2015). Intensification of Polychaete Worm Culture in Engineered Growth Systems. (Tesis Doctoral). Newcastle, UK: Newcastle University.

Moore, D. W., \& Dillon, T. M. (1993). The relationship between growth and reproduction in the marine polychaete Nereis (Neanthes) arenaceodentata (Moore): implications for chronic sublethal sediment bioassays. Journal of Experimental Marine Biology and Ecology, 173, 231-246.

Olive, P. J. W. (1994). Polychaeta as a word resource: a review of patterns of exploitation as sea angling baits and the potential for aquaculture based production. En J. C. Dauvin, L. Laubier, \& D. J. Reish (Eds.). Actes de la 4e'me Confrence Internationale des Polychètes (Vol. 162) (pp 593-602), Paris, France: Memoires du Musum National d' Histoire Naturelle.

Olive, P. J. W. (1999). Polychaete aquaculture and polychaete science: a mutual synergism. Hydrobiologia, 402, 175-183.

Pardo, E. V., Teixeira, L. L., \& Amaral, A. C. (2010). Morphometric analysis of Capitella capitata (Polychaeta, Capitellidae). Iheringia Série Zoologica, 100, 13-18.

Pires, A., Figueira, E., Moreira, A., Soares M. V. M. A., \& Freitas, R. (2015). The effects of water acidification, temperatura and salinity on the regenerative capacity of the polychaete Diopatra neapolitana. Marine Environmental Research, 106, 30-41.

Quian, P. Y., \& Chia, F. S. (1992). Effect of aging on reproduction in a marine polychaete Capitella sp. Journal of Experimental Marine Biology, 156, 23-38.

Prevedelli, D. (1991). Influence of temperatura and diet on survival of Perinereis rullieri pilato (Polychaeta, Nereididae). Italian Journal of Zoology, 58(3), 225-228.
Reish, D. J., \& Martin, J. M. (1976). The effect of heavy metals on laboratory populations of two polychaetes with comparisons to the water quality conditions and standards in Southern California marine waters. Water Research, 10, 299-302.

Reish, D., \& Gerlinger, T. (1997). A Review of the toxicological studies with Polychaetous Annelids. Bulletin of Marine Science, 60(2), 584-607.

Ryther, J. H., Goldman, J. C., Gifford, C. E., Huguenin, J. E., Wing, A. S., Clarner, P. J., ... LaPointe, B. E. (1975). Physical integrated waste-recycling marine polyculture systems. Aquaculture, 5, 163-177.

Silva, N., \& Guerra, D. (2008). Distribución de temperatura, salinidad, oxígeno disuelto y nutrientes en el canal Pulluche-Chacabuco, Chile. (Crucero CIMAR 9 fiordos). Ciencia y Tecnología del Mar, 31(2), 29-43.

Serebiah J. S. (2015). Culture of Marine Polychaetes. En S. Perumal, \& A. R. T. Pachiappan, (Eds.) Advances in Marine and Brackishwater Aquaculture (pp 43-49). New Delhi, India: Springer.

Snelgrove, V. R. P. (1999). Getting to the Bottom of Marine Biodiversity: Sedimentary Habitats. Ocean bottom are the most widespread habita ton Earth and support high biodiversity and key ecosystem services. BioScience, 49(2), 129-138.

Tomassetti, P., \& Porrello, S. (2005). Polychaetes as indicators of marine fish farm organic enrichment. Aquatics. International, 13, 109-128.

To-orn, N., \& Paphavasit, N. (2017). Bioremediation of organically enriched sediment under green mussel rafts using Spionids Genus Prionospio (Polychaeta: Spionidae). EnvironmentAsia, 10, 17-24.

Tsutsumi, H., \& Kikuchi, T. (1984). Study of the life history of Capitella capitata (Polychaeta: Capitellidae) in Amakusa, South Japan including a comparison with other geographical regions. Marine Biology, $80,315-321$.

Warren, L. M. (1976). A Population study of the polychaete Capitella capitata at Plymouth. Marine Biology, 38, 209-216. 